\title{
ANALYSIS AND CONVERGENCE OF THE MAC SCHEME. II. NAVIER-STOKES EQUATIONS
}

\author{
R. A. NICOLAIDES AND X. WU
}

\begin{abstract}
The MAC discretization scheme for the incompressible NavierStokes equations is interpreted as a covolume approximation to the equations. Using some results from earlier papers dealing with covolume error estimates for div-curl equation systems, and under certain conditions on the data and the solutions of the Navier-Stokes equations, we obtain first-order error estimates for both the vorticity and the pressure.
\end{abstract}

\section{INTRODUCTION}

In a previous paper [4], the classical MAC scheme for viscous fluid flows was analyzed. It was proved that the scheme is first-order accurate in a discrete $H^{1}$ norm when applied to the stationary Stokes equations. To our knowledge, this is the first such analysis for the stationary problem. Earlier, Porsching [7] had considered the (nonlinear) evolution problem and obtained certain estimates. These estimates contain factors which are exponentially increasing with time and so are not immediately relevant to the stationary case. In this paper we extend the results of [4] to the stationary Navier-Stokes equations in two dimensions under hypotheses which ensure that the equations have a unique solution.

The analysis which is presented below follows the general lines of [4] with allowance being made for the new difficulties caused by the nonlinear terms. Generally, this paper is written so as to be independent of [4]. The MAC method is derived as a special case of the covolume formulation in [3].

In writing out the Navier-Stokes equations there are several possibilities for the form of the nonlinear term. The main ones are the standard $\mathbf{u} \cdot \nabla \mathbf{u}$ form, the conservative form $\operatorname{div} \mathbf{u} \times \mathbf{u}$ and the total pressure form which in two space dimensions is $\mathbf{u}^{\prime} \omega$. We have chosen to present the analysis for the latter form. Our choice of the total pressure form is motivated in part by some recent numerical results [1], which show that at least in some cases it produces markedly superior results to the other two possibilities. The work in [6] contains some additional numerical results.

\section{Mesh notations}

Let $\Omega$ be a bounded rectangular domain in $R^{2}$ with boundary $\Gamma$. We will use a Cartesian mesh with $x$ - and $y$-spacing equal to $h$ and $h^{\prime}$. To avoid unnecessary

Received by the editor October 25, 1991 and, in revised form, December 2, 1993.

1991 Mathematics Subject Classification. Primary 76M25, 76D05, 65N12.

Key words and phrases. Covolume, MAC, Navier-Stokes.

This work was supported by the United States Air Force under grant AFOSR 90-0359. 
complications, we will assume that $h=h^{\prime}$. It is easy to modify the results to cover the contrary case. A staggered mesh is formed by connecting the centers of the rectangles (cells) to adjacent cell centers and to the midpoints of the boundary edges. The $N$ nodes of the primal mesh are numbered $1,2, \ldots, N$ in some suitable way, and the $T$ nodes of the dual mesh are similarly numbered $1,2, \ldots, T$. The $E$ edges (both primal and dual) are labeled $1,2, \ldots, E$ in some convenient way. The cells, edges and nodes of the primal mesh are denoted by $\tau_{i}, \sigma_{j}$ and $\nu_{k}$, respectively. Those of the dual mesh are similarly denoted by primed quantities such as $\sigma_{j}^{\prime}$. A direction is assigned to each primal edge according to the rule that positive is from low to high node number. The dual edges are directed by the convention that $\left(\sigma_{j}^{\prime}, \sigma_{j}\right)$ are oriented like the $(x, y)$ axes of the coordinate system.

Let $\mathbf{n}$ denote the normal direction of $\sigma_{j}$ directed along $\sigma_{j}^{\prime}$. The discrete equations use the normal velocity components defined at the midpoint of $\sigma_{j}$ as unknowns denoted by $u_{j}$. The set of normal velocity components defined on edges can be identified with $R^{E}$. We introduce an inner product into $R^{E}$ by

$$
(u, v)_{W}:=\sum_{\sigma_{j} \in \bar{\Omega}} u_{j} v_{j} h h_{j}^{\prime}
$$

where $h_{j}^{\prime}$ is the length of $\sigma_{j}^{\prime}$, where $h_{j}^{\prime}=h$ if $\sigma_{j}^{\prime}$ is an interior edge and $h / 2$ if $\sigma_{j}^{\prime}$ is on the boundary. The associated norm is denoted by $\|\cdot\|_{W}$. Clearly, it is twice a discrete $L^{2}$ norm. This inner product space is denoted by $\mathcal{U}$. We denote by $\mathcal{U}_{0}$ the space

$$
\mathcal{U}_{0}:=\left\{u \in \mathcal{U} ;\left.u\right|_{\Gamma}=0\right\} .
$$

We will also use a discrete $L^{p}$ norm; for $u \in R^{E}$ it is defined by

$$
\|u\|_{W, p}:=\left(\sum_{\sigma_{j} \in \bar{\Omega}}\left|u_{j}\right|^{p} h h_{j}^{\prime}\right)^{1 / p} .
$$

Scalar fields defined at the dual nodes $\nu_{i}^{\prime}$ can be identified with elements of $R^{T}$. An inner product on $R^{T}$ is defined by

$$
(\phi, \theta)_{A}:=\sum_{\tau_{i} \in \bar{\Omega}} \phi_{i} \theta_{i} A_{i},
$$

where $A_{i}$ denotes the area of the $i$ th cell. The associated norm is denoted by $\|\cdot\|_{A}$. This inner product space is denoted by $\mathcal{P}$. Similarly, scalar fields defined on the primal nodes $\nu_{k}$ can be identified with elements of $R^{N}$, and an inner product defined by

$$
(\psi, \chi)_{A^{\prime}}:=\sum_{\tau_{k}^{\prime} \in \bar{\Omega}} \psi_{k} \chi_{k} A_{k}^{\prime}
$$

where $A_{k}^{\prime}$ denotes the area of the $k$ th dual cell. The norm is denoted by $\|\cdot\|_{A^{\prime}}$, and the inner product space by $\mathcal{S}$, or by $\mathcal{S}_{0}$ if the boundary values are all zero.

For each primal cell $\tau_{i}$, discrete flux and divergence operators are defined on $\mathcal{U}$ by

$$
(\widehat{D} u)_{i}:=\sum_{\sigma_{j} \in \partial \tau_{i}} u_{j} \tilde{h}
$$


and

$$
(D u)_{i}:=(\widehat{D} u)_{i} / A_{i} .
$$

By $\tilde{h}$ we mean $h$ negatively signed if the corresponding velocity component is directed towards the inside of $\tau_{i}$, and positively signed otherwise.

For each interior dual cell $\tau_{k}^{\prime}$ discrete circulation and curl operators are defined by

$$
(\widehat{C} u)_{k}:=\sum_{\sigma_{j}^{\prime} \in \partial \tau_{k}^{\prime}} u_{j} \tilde{h}_{j}^{\prime}
$$

and

$$
(C u)_{k}:=(\widehat{C} u)_{k} / A_{k}^{\prime} .
$$

The tilde here on $h_{j}^{\prime}$ means a negative sign if the dual edge is directed against the positive sense of description of $\partial \tau_{k}^{\prime}$ and a positive sign otherwise.

Extensions of $C$ and $\widehat{C}$ to the boundary are denoted by $C_{b}$ and $\widehat{C}_{b}$. In this case, the velocity components along the boundary segments defined by the intersections of consecutive dual mesh edges with $\Gamma$ must be specified.

We also introduce an operator $R$ such that

$$
R \psi \in R^{E}, \quad(R \psi)_{j}:=\frac{\psi_{k_{2}}-\psi_{k_{1}}}{h} \quad \forall \psi \in R^{n},
$$

where the positive direction of $\sigma_{j}$ is from $\nu_{k_{1}}$ to $\nu_{k_{2}}$. An operator $G$ is defined such that

$$
G \phi \in R^{E}, \quad(G \phi)_{j}:=\frac{\phi_{i_{2}}-\phi_{i_{1}}}{h_{j}^{\prime}} \quad \forall \phi \in R^{T},
$$

where the positive direction of $\sigma_{j}^{\prime}$ is from $\nu_{i_{1}}^{\prime}$ to $\nu_{i_{2}}^{\prime}$.

When we deal with the approximations for certain items, we need to map the cell, edge, or kite (the parallelogram with $\sigma_{j}$ and $\sigma_{j}^{\prime}$ as diagonals) to a standard one. We will use $B$ to denote the mappings and a hat to denote the standard cell, edge, or kite. For example, $\hat{\tau}^{\prime}(-1 / 2 \leq \hat{x} \leq 1 / 2,-1 / 2 \leq \hat{y} \leq 1 / 2)$ is the image of $\tau_{k}^{\prime}$.

\section{Discretization of Navier-Stokes Equations}

We consider the incompressible Navier-Stokes equations,

$$
\begin{array}{rlrl}
-\nu \Delta \mathbf{u}+(\mathbf{u} \cdot \nabla) \mathbf{u}+\nabla p & =\mathbf{f} & & \text { in } \Omega, \\
\operatorname{div} \mathbf{u} & =0 & & \text { in } \Omega, \\
\left.\mathbf{u}\right|_{\Gamma} & =0 . &
\end{array}
$$

We will discretize (1) in the form

$$
-\nu \Delta \mathbf{u}-\mathbf{u}^{\prime} \omega+\nabla\left(p+\frac{|\mathbf{u}|^{2}}{2}\right)=\mathbf{f},
$$

where $\omega=$ curl $\mathbf{u}$ and $\mathbf{u}^{\prime}=\left(-u_{2}, u_{1}\right) ; u_{1}$ and $u_{2}$ are the two velocity components of $\mathbf{u}$. This form is often used in practice, and there is evidence that it can give better results than (1) or its momentum conservation form $(\operatorname{div} \mathbf{u} \times \mathbf{u})$. 


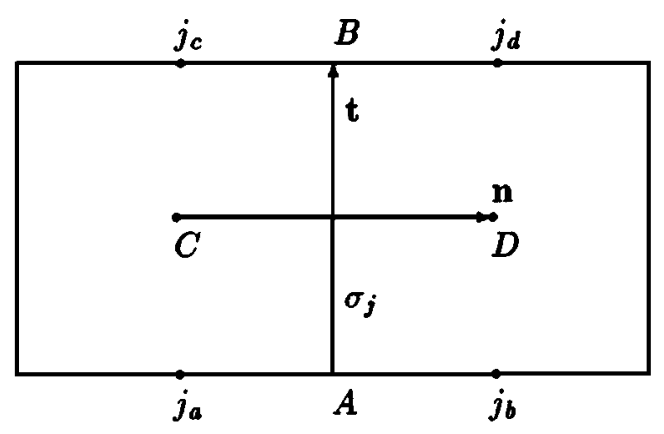

FiguRE 1

Figure 1 shows two adjacent mesh cells, sharing the common edge $\sigma_{j}(A B)$ and with dual edge $\sigma_{j}^{\prime}(C D)$. Let $\mathbf{n}$ denote the positive direction of the $\sigma_{j}^{\prime}$. Taking the dot product with $\mathbf{n}$ on both sides of (4), we get by a calculation

$$
\nu \frac{\partial}{\partial t}(\operatorname{curl} \mathbf{u})-\nu \frac{\partial}{\partial n}(\operatorname{div} \mathbf{u})-\mathbf{u} \cdot \mathbf{t} \omega+\frac{\partial \hat{p}}{\partial n}=\mathbf{f} \cdot \mathbf{n},
$$

where $\mathbf{t}$ is the positive direction of $\sigma_{j}(A B)$ as shown in Figure 1 and $\hat{p}=p+|\mathbf{u}|^{2} / 2$. For convenience, we will denote the total pressure $\hat{p}$ by $p$ from now on. We have left the divergence term in (5), even though according to (2) it is zero. There are two reasons why we feel that allowing this term to remain is significant. First, it reveals an important symmetry between the circulation and flux operator, in that they are seen to be analogous to the real and imaginary parts of a complex derivative. Second, by carrying the flux term through the analysis we are able to show how it may be estimated. Such estimates would be necessary if a nonzero divergence was prescribed or in circumstances when the divergence constraint is not met exactly.

Integrating (5) over the primal edge $\sigma_{j}$, we get

$$
\begin{aligned}
\nu \frac{\omega(B)-\omega(A)}{h} & -\frac{1}{h} \int_{\sigma_{j}} \nu \frac{\partial(\operatorname{div} \mathbf{u})}{\partial n} d s \\
& -\frac{1}{h} \int_{\sigma_{j}} \mathbf{u} \cdot \mathbf{t} \omega d s+\frac{1}{h} \int_{\sigma_{j}} \frac{\partial p}{\partial n} d s=\frac{1}{h} \int_{\sigma_{j}} \mathbf{f} \cdot \mathbf{n} d s
\end{aligned}
$$

where the positive direction is from $A$ to $B$. For the discretization of this equation we replace $\omega(A)$ and $\omega(B)$ by the discrete curls $\omega_{A}^{\prime}$ and $\omega_{B}^{\prime}$ at the nodes, approximate the normal derivative of $\operatorname{div} \mathbf{u}$ by the finite difference of the discrete divs $(D u)_{C}$ and $(D u)_{D}$ at the centers $C$ and $D$ and use the average of $\omega_{A}^{\prime}$ and $\omega_{B}^{\prime}$ to approximate the vorticity in the integral. Then we have

$$
\nu \frac{\omega_{B}^{\prime}-\omega_{A}^{\prime}}{h}-\nu \frac{(D u)_{D}-(D u)_{C}}{h}-u_{j}^{t} \frac{\left(\omega_{B}^{\prime}+\omega_{A}^{\prime}\right)}{2}+\frac{p_{D}^{\prime}-p_{C}^{\prime}}{h}=f_{j},
$$

where $p_{C}^{\prime}$ and $p_{D}^{\prime}$ are the discrete pressure defined at the centers of the primal cells, $f_{j}$ is the right-hand side of (6) and $u_{j}^{t}$ is the tangential component computed as

$$
u_{j}^{t}:=\frac{1}{4}\left(\tilde{u}_{j_{a}}+\tilde{u}_{j_{b}}+\tilde{u}_{j_{c}}+\tilde{u}_{j_{d}}\right) .
$$


The tilde here means a positive sign if the corresponding dual edge $\left(\sigma_{j_{a}}^{\prime}, \ldots, \sigma_{j_{d}}^{\prime}\right)$ has the same direction as $\sigma_{j}$ and a negative sign otherwise. Note that

$$
\left\|u^{t}\right\|_{W, p} \leq\|u\|_{W, p} \text { for } 2 \leq p<\infty,
$$

where $u^{t} \in R^{E}$ is the tangential vector with $u_{j}^{t}$ as components.

The discretization of the incompressibility condition and the boundary condition are similar; we have

$$
\begin{aligned}
& D u=0, \\
& \left.u\right|_{\Gamma}=\left.u^{t}\right|_{\Gamma}=0 .
\end{aligned}
$$

Equation (7) contains a term $\omega_{B}^{\prime}+\omega_{A}^{\prime}$ for each primal edge. Regarded as an element of $R^{E}$, the $W$ norm of this vector can be estimated by

$$
\left\|\omega_{B}^{\prime}+\omega_{A}^{\prime}\right\|_{W} \leq\left\|\omega_{B}^{\prime}\right\|_{W}+\left\|\omega_{A}^{\prime}\right\|_{W} .
$$

The following lemma gives a relation between the $W$ norm and $A^{\prime}$ norm of this vector.

Lemma 1. Let $\omega_{B}^{\prime}$ and $\omega_{A}^{\prime}$ denote the $R^{E}$ vectors which take the values of $\omega^{\prime}$ at the nodes corresponding to the positive and negative direction of the edges, respectively. Then

$$
\left\|\omega_{B}^{\prime}\right\|_{W}^{2}+\left\|\omega_{A}^{\prime}\right\|_{W}^{2}=4\left\|\omega^{\prime}\right\|_{A^{\prime}}^{2}
$$

Proof. Let $E_{j}(B)$ and $E_{j}(A)$ denote the sets of primal edges with node $\nu_{k}$ as their high and low node numbers respectively. Then

$$
\omega_{k}^{\prime 2} \sum_{E_{j}(B)} h h_{j}^{\prime}+\omega_{k}^{\prime 2} \sum_{E_{j}(A)} h h_{j}^{\prime}=\omega_{k}^{\prime 2} \sum_{E_{j}} h h_{j}^{\prime}=4 \omega_{k}^{\prime 2} A_{k}^{\prime},
$$

where $E_{j}$ is the set of primal edges connected to node $\nu_{k}$. Taking the summation over all nodes, we get (12).

Lemma 2. Assume $u \in \mathcal{U}_{0}$ satisfies the equations

$$
\begin{aligned}
& C u=\omega^{\prime}, \\
& D u=g, \quad \sum_{i} g_{i} A_{i}=0, \\
& \left.u\right|_{\Gamma}=0
\end{aligned}
$$

and $2 \leq p<\infty$. Then there exists a constant $\sigma$ independent of $h$ such that

$$
\|u\|_{W, p} \leq \sigma\left(\left\|\omega^{\prime}\right\|_{A^{\prime}}+\|g\|_{A}\right) .
$$

Proof. Define a piecewise constant function with values $\omega_{k}^{\prime}$ in interior dual cells $\tau_{k}^{\prime}$ and zero in the boundary dual cells. We will use the same notation $\omega^{\prime}$ for this function as for its values. Define a piecewise constant function which is a constant $g_{i}$ in cell $\tau_{i}$ and also denote this function by $g$. Consider the following problem:

$$
\begin{aligned}
\operatorname{curl} \mathbf{u}^{*} & =\omega^{\prime}, \\
\operatorname{div} \mathbf{u}^{*} & =g, \quad \int_{\Omega} g d x d y=0, \\
\left.\mathbf{u}^{*} \cdot \mathbf{n}\right|_{\Gamma} & =0 .
\end{aligned}
$$


Define $u^{*}$ by

$$
u_{j}^{*}=\frac{1}{h} \int_{\sigma_{j}} \mathbf{u}^{*} \cdot \mathbf{n} d s
$$

Then by [5, Theorem 6.1] we have

$$
\left\|u-u^{*}\right\|_{W} \leq K h\left|\mathbf{u}^{*}\right|_{1, \Omega} .
$$

Here and below, $K$ denotes a generic constant. It follows that

$$
\begin{aligned}
\left\|u-u^{*}\right\|_{W, p}^{p} & =\sum_{\sigma_{j} \in \bar{\Omega}}\left(u_{j}-u_{j}^{*}\right)^{p} h h_{j}^{\prime} \\
& \leq K\left(\sum_{\sigma_{j} \in \bar{\Omega}}\left(u_{j}-u_{j}^{*}\right)^{2} h h_{j}^{\prime} h^{4 / p-2}\right)^{p / 2} \\
& =K h^{2-p}\left\|u-u^{*}\right\|_{W}^{p} .
\end{aligned}
$$

Thus,

$$
\begin{aligned}
\left\|u-u^{*}\right\|_{W, p} & \leq h^{2 / p-1}\left\|u-u^{*}\right\|_{W} \\
& \leq K h^{2 / p}\left|\mathbf{u}^{*}\right|_{1, \Omega}
\end{aligned}
$$

Define $\bar{u}^{*} \in \mathcal{U}$ by

$$
\bar{u}_{j}^{*}:=\frac{1}{\left|\kappa_{j}\right|} \int_{\kappa_{j}} \mathbf{u}^{*} \cdot \mathbf{n} d x d y
$$

where $\kappa_{j}$ is the kite area associated with $\sigma_{j}$ and $\sigma_{j}^{\prime}$. By mapping $\kappa_{j}$ to a standard kite $\hat{\kappa}$, we can see that $\bar{u}_{j}^{*}-u_{j}^{*}$ is bounded on $\mathbf{H}^{1}(\hat{\kappa})$ and vanishes for constant functions. Then from standard approximation theory we have the estimate

$$
\left\|\bar{u}^{*}-u^{*}\right\|_{W} \leq K h\left|\mathbf{u}^{*}\right|_{1, \Omega}
$$

and therefore

$$
\left\|\bar{u}^{*}-u^{*}\right\|_{W, p} \leq K h^{2 / p}\left|\mathbf{u}^{*}\right|_{1, \Omega} .
$$

On the other hand,

$$
\begin{aligned}
\left|\frac{1}{\left|\kappa_{j}\right|} \int_{\kappa_{j}} \mathbf{u}^{*} \cdot \mathbf{n} d x d y\right| & =\left|\frac{1}{|\hat{\kappa}|} \int_{\hat{\kappa}} \hat{\mathbf{u}}^{*} \cdot \hat{\mathbf{n}} d \hat{x} d \hat{y}\right| \\
& \leq K\left(\int_{\hat{\kappa}}\left|\hat{\mathbf{u}}^{*}\right|^{p}\right)^{1 / p} \\
& \leq K h^{-2 / p}\left\|\mathbf{u}^{*}\right\|_{0, p, \kappa_{j}}
\end{aligned}
$$

and then

$$
\begin{aligned}
\left\|\bar{u}^{*}\right\|_{W, p} & \leq K\left(\sum_{j} h^{-2}\left\|\mathbf{u}^{*}\right\|_{0, p, \kappa_{j}}^{p} h^{2}\right)^{1 / p} \\
& \leq K\left\|\mathbf{u}^{*}\right\|_{0, p, \Omega} \\
& \leq K\left\|\mathbf{u}^{*}\right\|_{1, \Omega} .
\end{aligned}
$$


Combining (13), (14) and (15), we get

$$
\begin{aligned}
\|u\|_{W, p} & \leq\left\|u-u^{*}\right\|_{W, p}+\left\|u^{*}-\bar{u}^{*}\right\|_{W, p}+\left\|\bar{u}^{*}\right\|_{W, p} \\
& \leq K\left\|\mathbf{u}^{*}\right\|_{1, \Omega} .
\end{aligned}
$$

Since $\mathbf{u}^{*} \cdot \mathbf{n}$ vanishes on the boundary, $\left\|\operatorname{curl} \mathbf{u}^{*}\right\|_{0, \Omega}+\left\|\operatorname{div} \mathbf{u}^{*}\right\|_{0, \Omega}$ is a norm for $\mathbf{H}^{1}(\Omega)$ and is equivalent to norm $\left\|\mathbf{u}^{*}\right\|_{1, \Omega}$ (see e.g. [2]). Therefore

$$
\begin{aligned}
\|u\|_{W, p} & \leq K\left(\left\|\operatorname{curl} \mathbf{u}^{*}\right\|_{0, \Omega}+\left\|\operatorname{div} \mathbf{u}^{*}\right\|_{0, \Omega}\right) \\
& \leq \sigma\left(\left\|\omega^{\prime}\right\|_{A^{\prime}}+\|g\|_{A}\right),
\end{aligned}
$$

where $\sigma$ is a constant independent of $h$.

Theorem 1. Let $\tilde{\sigma}=\max \left\{1, \sigma^{2}\right\}$, and assume that the function $f$ and the viscosity $\nu$ are such that

$$
\frac{16 \sigma \tilde{\sigma}\|f\|_{W}}{\nu^{2}} \leq 1 .
$$

Then the discrete Navier-Stokes equations (7), (10) and (11) have a solution (u, $\left.p^{\prime}\right)$. Here, $u$ is unique and $p^{\prime}$ is unique apart from an additive constant.

Proof. Introduce a space $\mathcal{V}$ defined by

$$
\mathcal{V}:=\left\{v \in \mathcal{U}_{0} ; D v=0\right\}
$$

and a trilinear form $a_{1}(u ; v, w)$ defined by

$$
a_{1}(u ; v, w):=\sum_{\sigma_{j} \in \bar{\Omega}} u_{j} v_{j} w_{j} h h_{j}^{\prime} .
$$

Taking the inner product of $v \in \mathcal{V}$ with equation (7) and using summation by parts, we have

$$
\nu\left(v, R \omega^{\prime}\right)_{W}-a_{1}\left(u^{t} ; v, M \omega^{\prime}\right)=(v, f)_{W} \quad \forall v \in \mathcal{V},
$$

where the operator $M$ is defined by $\left(M \omega^{\prime}\right)_{j}:=\left(\omega_{B}^{\prime}+\omega_{A}^{\prime}\right) / 2$.

For the existence of the discrete velocity $u$, let $u^{m} \in \mathcal{V}$ be given; we look for $u^{m+1} \in \mathcal{V}$ satisfying the iteration scheme

$$
\nu\left(v, R \omega^{\prime, m+1}\right)_{W}-a_{1}\left(u^{t, m} ; v, M \omega^{\prime, m+1}\right)=(v, f)_{W} \quad \forall v \in \mathcal{V} .
$$

It is easy to show that there is a unique $u^{m+1}$ satisfying this iteration scheme. Take $v=u^{m+1} ;$ we have

$$
\nu\left(u^{m+1}, R \omega^{\prime, m+1}\right)_{W}-a_{1}\left(u^{t, m} ; u^{m+1}, M \omega^{\prime, m+1}\right)=\left(u^{m+1}, f\right)_{W}
$$

and from Lemmas 1 and 2 we get

$$
\begin{aligned}
\nu\left\|\omega^{\prime, m+1}\right\|_{A^{\prime}}^{2} & \leq\left\|M \omega^{\prime, m+1}\right\|_{W}\left\|u^{m+1} u^{t, m}\right\|_{W}+\left\|u^{m+1}\right\|_{W}\|f\|_{W} \\
& \leq 2\left\|\omega^{\prime, m+1}\right\|_{A^{\prime}}\left\|u^{m+1} u^{t, m}\right\|_{W}+\sigma\left\|\omega^{\prime, m+1}\right\|_{A^{\prime}}\|f\|_{W} .
\end{aligned}
$$

By (9) and Lemma 2 with $p=4$ this leads to

$$
\begin{aligned}
\nu\left\|\omega^{\prime, m+1}\right\|_{A^{\prime}} & \leq 2\left\|u^{m+1} u^{t, m}\right\|_{W}+\sigma\|f\|_{W} \\
& \leq 2\left\|u^{m+1}\right\|_{W, 4}\left\|u^{t, m}\right\|_{W, 4}+\sigma\|f\|_{W} \\
& \leq 2\left\|u^{m+1}\right\|_{W, 4}\left\|u^{m}\right\|_{W, 4}+\sigma\|f\|_{W} \\
& \leq 2 \sigma\left\|\omega^{\prime, m+1}\right\|_{A^{\prime}}\left\|\omega^{\prime, m}\right\|_{A^{\prime}}+\sigma\|f\|_{W}
\end{aligned}
$$


or

$$
\left\|\omega^{\prime, m+1}\right\|_{A^{\prime}} \leq \frac{\sigma\|f\|_{W}}{\nu-2 \sigma\left\|\omega^{\prime, m}\right\|_{A^{\prime}}} .
$$

Taking $\omega^{\prime, 0}$ such that $\left\|\omega^{\prime, 0}\right\|_{A^{\prime}} \leq 2 \sigma\|f\|_{W} / \nu$ we conclude that

$$
\left\|\omega^{\prime, m+1}\right\|_{A^{\prime}} \leq \frac{2 \sigma\|f\|_{W}}{\nu}
$$

Since $\omega^{\prime, m}$ is bounded, by Lemma 2 with $p=2$ we know that the sequence $\left\{u^{m}\right\}$ is also bounded. Since $\left\{u^{m}\right\}$ is a sequence in $R^{E}$, we may extract a subsequence $\left\{u^{m_{s}}\right\}$ which converges to $u$ in $R^{E}$. Therefore $\omega^{\prime, m}$ converges to $\omega^{\prime}=C_{b} u$ and $u$ satisfies

$$
\nu\left(C_{b} v, C_{b} u\right)_{W}-a_{1}\left(u^{t} ; v, M \omega^{\prime}\right)=(v, f)_{W} \quad \forall v \in \mathcal{V}
$$

By taking the limit in (17) we get a bound for $\omega^{\prime}$ :

$$
\left\|\omega^{\prime}\right\|_{A^{\prime}} \leq \frac{2 \sigma\|f\|_{W}}{\nu}
$$

To prove the uniqueness, let $\tilde{u}$ be another solution satisfying (18) and (19) and define $\varepsilon:=u-\tilde{u}$. By taking the difference of the two equations and setting $v=\varepsilon$, we have

$$
\nu\left(C_{b} \varepsilon, C_{b} \varepsilon\right)_{W}-a_{1}\left(u^{t} ; \varepsilon, M \omega^{\prime}\right)+a_{1}\left(\tilde{u}^{t} ; \varepsilon, M \tilde{\omega}^{\prime}\right)=0 .
$$

Then using Lemmas 1 and 2, we get

$$
\begin{aligned}
\nu\left\|C_{b} \varepsilon\right\|_{A^{\prime}}^{2} & \leq a_{1}\left(u^{t} ; \varepsilon, M\left(\omega^{\prime}-\tilde{\omega}^{\prime}\right)\right)+a_{1}\left(u^{t}-\tilde{u}^{t} ; \varepsilon, M \tilde{\omega}^{\prime}\right) \\
& \leq 2\left\|\omega^{\prime}-\tilde{\omega}^{\prime}\right\|_{A^{\prime}}\left\|u^{t} \varepsilon\right\|_{W}+2\left\|\tilde{\omega}^{\prime}\right\|_{A^{\prime}}\left\|\varepsilon\left(u^{t}-\tilde{u}^{t}\right)\right\|_{W} \\
& \leq 2\left\|C_{b} \varepsilon\right\|_{A^{\prime}}\|\varepsilon\|_{W, 4}\left\|u^{t}\right\|_{W, 4}+2\left\|\tilde{\omega}^{\prime}\right\|_{A^{\prime}}\|\varepsilon\|_{W, 4}\left\|u^{t}-\tilde{u}^{t}\right\|_{W, 4} \\
& \leq 2 \sigma\left\|C_{b} \varepsilon\right\|_{A^{\prime}}^{2}\|u\|_{W, 4}+2 \sigma\left\|\tilde{\omega}^{\prime}\right\|_{A^{\prime}}\left\|C_{b} \varepsilon\right\|_{A^{\prime}}\|u-\tilde{u}\|_{W, 4} \\
& \leq 2 \sigma^{2}\left\|C_{b} \varepsilon\right\|_{A^{\prime}}^{2}\left(\left\|\omega^{\prime}\right\|_{A^{\prime}}+\left\|\tilde{\omega}^{\prime}\right\|_{A^{\prime}}\right) \\
& \leq \frac{\nu}{2}\left\|C_{b} \varepsilon\right\|_{A^{\prime}}^{2}
\end{aligned}
$$

which implies $\left\|C_{b} \varepsilon\right\|_{A^{\prime}}=0$ and therefore $C_{b} \varepsilon=0$. Since $D \varepsilon=0$, by [5, Theorem 5.3] we have $u=\tilde{u}$.

We now prove the existence of the discrete pressure $p^{\prime}$. Let

$$
r_{j}:=\nu\left(R \omega^{\prime}\right)_{j}-u_{j}^{t}\left(M \omega^{\prime}\right)_{j}-f_{j}, \quad j=1, \ldots, E .
$$

Since $(r, v)_{W}=0$ for $v \in \mathcal{V}$, we have that $r$ is orthogonal to $\mathcal{V}$. By [5, Theorem 5.3] we have

$$
\mathcal{U}_{0}=\mathcal{V} \oplus \mathcal{W}
$$

where $\mathcal{W}=\left\{u \in \mathcal{U}_{0}, C u=0\right\}$. Thus, $r \in \mathcal{W}$, and there exists a $p^{\prime} \in R^{T}$ such that $r=G p^{\prime}\left(\left[5\right.\right.$, Theorem 5.1]). Here, $p^{\prime}$ is unique up to a constant. 


\section{VORTICITY ERROR ESTIMATE}

We will need the following lemma for estimating the vorticity error.

Lemma 3. Assume that $\mathbf{u} \in \mathbf{H}^{2}(\Omega)$ and $\omega \in H^{2}(\Omega)$. Let $\omega_{a}$ and $\omega_{b}$ denote the values of $\omega$ defined at arbitrary points $P_{k}, Q_{k} \in \tau_{k}^{\prime}\left(\right.$ or $\left.\kappa_{j}\right)$. Then for $h$ small enough we have

$$
\left\|\omega_{a}-\omega_{b}\right\|_{A^{\prime}} \leq K h|\mathbf{u}|_{2, \Omega}
$$

and

$$
\left\|\omega_{a}-\omega_{b}\right\|_{W} \leq K h|\mathbf{u}|_{2, \Omega} .
$$

Proof. Let $\mathbf{s}_{k}$ denote the unit vector in the direction of the segment $r_{k}=P_{k} Q_{k}$, and let $\hat{r}$ denote the image of $r_{k}$ in $\hat{\tau}^{\prime}$. Then we have

$$
\begin{aligned}
\left|\omega\left(Q_{k}\right)-\omega\left(P_{k}\right)\right| & =\left|\int_{P_{k}}^{Q_{k}} \frac{\partial \omega}{\partial s_{k}} d s\right| \\
& \leq(2 h)^{1 / 2}\left\|\frac{\partial \omega}{\partial s_{k}}\right\|_{0, r_{k}} \\
& \leq K\left\|\frac{\partial \hat{\omega}}{\partial \hat{s}_{k}}\right\|_{0, \hat{r}} \\
& \leq K\left\|\frac{\partial \hat{\omega}}{\partial \hat{s}_{k}}\right\|_{1, \hat{\tau}^{\prime}} \\
& \leq K\left(|\omega|_{1, \tau_{k}^{\prime}}+h|\omega|_{2, \tau_{k}^{\prime}}\right) .
\end{aligned}
$$

It follows that

$$
\sum_{\tau_{k}^{\prime} \in \bar{\Omega}}\left|\omega\left(Q_{k}\right)-\omega\left(P_{k}\right)\right|^{2} h^{2} \leq K h^{2}\left(|\omega|_{1, \Omega}^{2}+h^{2}|\omega|_{2, \Omega}^{2}\right) .
$$

Now choose $h$ such that $h|\omega|_{2, \Omega} \leq|\omega|_{1, \Omega}$ to obtain

$$
\left(\sum_{\tau_{k}^{\prime} \in \bar{\Omega}}\left|\omega\left(Q_{k}\right)-\omega\left(P_{k}\right)\right|^{2} A_{k}^{\prime}\right)^{1 / 2} \leq K h|\mathbf{u}|_{2, \Omega},
$$

where $K$ is a constant independent of $h$. The other estimate is similar.

Theorem 2. Under the hypotheses of Theorem 1, and assuming that $\mathbf{u} \in \mathbf{H}^{2}(\Omega)$, $\omega \in H^{2}(\Omega)$ and

$$
\bar{\sigma}=\frac{4 \sigma^{3}}{\nu^{2}}\|f\|_{W}+\frac{2 \sigma\|\mathbf{u}\|_{C(\Omega)}}{\nu}<1
$$

the following estimate holds:

$$
\left\|\omega^{\prime}-\bar{\omega}\right\|_{A^{\prime}} \leq K(\mathbf{u}, p, \mathbf{f}, \nu) h
$$

where $\bar{\omega} \in R^{N}$ is the average of the vorticity over the dual cells, $\|\cdot\|_{C(\Omega)}$ is the usual sup norm and the constant $K$ depends on $\|\mathbf{u}\|_{2, \Omega},|p|_{2, \Omega},\|f\|_{W}$, and $\nu$ but not on $h$. 
Proof. The exact solution of the Navier-Stokes equations satisfies

$$
\begin{aligned}
\operatorname{curl} \mathbf{u} & =\omega, \\
\operatorname{div} \mathbf{u} & =0, \\
\left.\mathbf{u} \cdot \mathbf{n}\right|_{\Gamma} & =0
\end{aligned}
$$

The discrete covolume solutions of the Navier-Stokes equations satisfy

$$
\begin{aligned}
C_{b} u & =\omega^{\prime}, \\
D u & =0, \\
\left.u\right|_{\Gamma} & =0 .
\end{aligned}
$$

Let $u^{(1)}$ be defined by

$$
u_{j}^{(1)}:=\frac{1}{h} \int_{\sigma_{j}} \mathbf{u} \cdot \mathbf{n} d s .
$$

For convenience, we use the following notations:

$$
\begin{aligned}
\mu\left(\frac{\partial p}{\partial n}\right) & \in R^{E}, \quad\left(\mu\left(\frac{\partial p}{\partial n}\right)\right)_{j}:=\frac{1}{h} \int_{\sigma_{j}} \frac{\partial p}{\partial n} d s, \\
Z & \in R^{E}, \quad Z_{j}:=\frac{1}{h} \int_{\sigma_{j}} \mathbf{u} \cdot \mathbf{t} \omega d s \\
Z^{\prime} & \in R^{E}, \quad Z_{j}^{\prime}:=u_{j}^{t}\left(M \omega^{\prime}\right)_{j} .
\end{aligned}
$$

Taking the difference of equation (6) and equation (7), we get

$$
\nu\left(R\left(\omega-\omega^{\prime}\right)\right)_{j}-\left(Z_{j}-Z_{j}^{\prime}\right)+\left(\mu\left(\frac{\partial p}{\partial n}\right)\right)_{j}-\left(G p^{\prime}\right)_{j}=0 .
$$

Let $\varepsilon:=u^{(1)}-u$ and assume that $u^{(1)}$ has zero tangential components on $\Gamma$. We have

$$
\nu R C_{b} \varepsilon=Z-Z^{\prime}-\mu\left(\frac{\partial p}{\partial n}\right)+G p^{\prime}-\nu R\left(\omega-C_{b} u^{(1)}\right),
$$

and taking the inner product with $\varepsilon$, we conclude that

$$
\begin{aligned}
\nu\left(\varepsilon, R C_{b} \varepsilon\right)_{W}= & \left(\varepsilon, Z-Z^{\prime}\right)_{W}+\left(\varepsilon, G p^{\prime}\right)_{W}-\left(\varepsilon, \mu\left(\frac{\partial p}{\partial n}\right)\right)_{W} \\
& -\nu\left(\varepsilon, R\left(\omega-C_{b} u^{(1)}\right)\right)_{W} .
\end{aligned}
$$

Using summation by parts, and since $D \varepsilon=0$, we have

$$
\begin{aligned}
\nu\left\|C_{b} \varepsilon\right\|_{A^{\prime}}^{2} & =\left(\varepsilon, Z-Z^{\prime}\right)_{W}-\left(\varepsilon, \mu\left(\frac{\partial p}{\partial n}\right)-G \bar{p}\right)_{W}-\nu\left(C_{b} \varepsilon, \omega-C_{b} u^{(1)}\right)_{A^{\prime}} \\
& \leq\left|\left(\varepsilon, Z-Z^{\prime}\right)_{W}\right|+\|\varepsilon\|_{W}\left\|\mu\left(\frac{\partial p}{\partial n}\right)-G \bar{p}\right\|_{W}+\nu\left\|C_{b} \varepsilon\right\|_{A^{\prime}}\left\|\omega-C_{b} u^{(1)}\right\|_{A^{\prime}} \\
& \leq\left|\left(\varepsilon, Z-Z^{\prime}\right)_{W}\right|+\left\|C_{b} \varepsilon\right\|_{A^{\prime}}\left(\sigma\left\|\mu\left(\frac{\partial p}{\partial n}\right)-G \bar{p}\right\|_{W}+\nu\left\|\omega-C_{b} u^{(1)}\right\|_{A^{\prime}}\right),
\end{aligned}
$$

where $\bar{p}$ is the average of $p$ over the primal cells defined by

$$
\left.\bar{p}\right|_{\tau_{i}}:=\frac{1}{A_{i}} \int_{\tau_{i}} p d x d y .
$$


For the nonlinear term, we have

$$
\begin{aligned}
\left(\varepsilon, Z-Z^{\prime}\right)_{W}= & \sum_{\sigma_{j} \in \bar{\Omega}} \varepsilon_{j}\left(\frac{1}{h} \int_{\sigma_{j}} \mathbf{u} \cdot \mathbf{t} \omega d s-u_{j}^{t}\left(M \omega^{\prime}\right)_{j}\right) h h_{j}^{\prime} \\
= & \sum_{\sigma_{j} \in \bar{\Omega}} \varepsilon_{j} \frac{1}{h} \int_{\sigma_{j}} \mathbf{u} \cdot \mathbf{t}\left(\omega-\left(M \omega^{\prime}\right)_{j}\right) d s h h_{j}^{\prime} \\
& +\sum_{\sigma_{j} \in \bar{\Omega}} \varepsilon_{j} \frac{1}{h} \int_{\sigma_{j}}\left(\mathbf{u} \cdot \mathbf{t}-u_{j}^{t}\right)\left(M \omega^{\prime}\right)_{j} d s h h_{j}^{\prime} \\
= & : I_{1}+I_{2} .
\end{aligned}
$$

For the first term we have

$$
\begin{aligned}
& I_{1}= \sum_{\sigma_{j} \in \bar{\Omega}} \varepsilon_{j} \frac{1}{h} \int_{\sigma_{j}} \mathbf{u} \cdot \mathbf{t}\left(\omega-\left(M \omega^{\prime}\right)_{j}\right) d s h h_{j}^{\prime} \\
& \leq\|\varepsilon\|_{W}\left(\sum_{\sigma_{j} \in \bar{\Omega}}\left(\frac{1}{h} \int_{\sigma_{j}} \mathbf{u} \cdot \mathbf{t}\left(\omega-\left(M \omega^{\prime}\right)_{j}\right) d s\right)^{2} h h_{j}^{\prime}\right)^{1 / 2} \\
& \leq\|\varepsilon\|_{W}\|\mathbf{u}\|_{C(\Omega)}\left(\sum_{\sigma_{j} \in \bar{\Omega}}\left(\frac{1}{h} \int_{\sigma_{j}}\left|\omega-\left(M \omega^{\prime}\right)_{j}\right| d s\right)^{2} h h_{j}^{\prime}\right)^{1 / 2} \\
& \quad\left\{\left(\sum_{\sigma_{j} \in \bar{\Omega}}\left(\frac{1}{h} \int_{\sigma_{j}}\left|\omega-(M \omega)_{j}\right| d s\right)^{2} h h_{j}^{\prime}\right)^{1 / 2}\right. \\
&+\left(\sum_{\sigma_{j} \in \bar{\Omega}}\left(\frac{1}{h} \int_{\sigma_{j}}\left|\left(M \omega-M\left\|_{W}\right\| \mathbf{u} \|_{C(\Omega)} u^{(1)}\right)_{j}\right| d s\right)^{2} h h_{j}^{\prime}\right)^{1 / 2} \\
&\left.+\left(\sum_{\sigma_{j} \in \bar{\Omega}}\left(\frac{1}{h} \int_{\sigma_{j}}\left|\left(M C_{b} u^{(1)}-M \omega^{\prime}\right)_{j}\right| d s\right)^{2} h h_{j}^{\prime}\right)^{1 / 2}\right\} \\
&=\|\varepsilon\|_{W}\|\mathbf{u}\|_{C(\Omega)}\left(\sum_{\sigma_{j} \in \bar{\Omega}}\left(\frac{1}{h} \int_{\sigma_{j}}\left|\omega-(M \omega)_{j}\right| d s\right)^{2} h h_{j}^{\prime}\right)^{1 / 2} \\
&+\|\varepsilon\|_{W}\|\mathbf{u}\|_{C(\Omega)}\left(\left\|M\left(\omega-C_{b} u^{(1)}\right)\right\|_{W}+\left\|M\left(C_{b} u^{(1)}-\omega^{\prime}\right)\right\|_{W}\right),
\end{aligned}
$$

where $\|\cdot\|_{C(\Omega)}$ is the usual sup norm. Since $\omega \in H^{2}(\Omega)$, there exists a point $P_{j} \in \sigma_{j}$ such that

$$
(M \omega)_{j}=\omega\left(P_{j}\right),
$$

and therefore there exists another point $Q_{j} \in \sigma_{j}$ such that

$$
\frac{1}{h} \int_{\sigma_{j}}\left|\omega-(M \omega)_{j}\right| d s=\left|\omega\left(Q_{j}\right)-\omega\left(P_{j}\right)\right| .
$$


Then from Lemma 3 we get

$$
\left(\sum_{\sigma_{j} \in \bar{\Omega}}\left(\frac{1}{h} \int_{\sigma_{j}}\left|\omega-(M \omega)_{j}\right| d s\right)^{2} h h_{j}^{\prime}\right)^{1 / 2} \leq K h|\mathbf{u}|_{2, \Omega},
$$

where $K$ is a constant independent of $h$. Hence, we have the estimate for $I_{1}$ :

$$
\begin{aligned}
I_{1} & \leq\|\varepsilon\|_{W}\|\mathbf{u}\|_{C(\Omega)}\left(K h|\mathbf{u}|_{2, \Omega}+\left\|M\left(\omega-C_{b} u^{(1)}\right)\right\|_{W}+\left\|M\left(C_{b} u^{(1)}-\omega^{\prime}\right)\right\|_{W}\right) \\
& \leq \sigma\left\|C_{b} \varepsilon\right\|_{A^{\prime}}\|\mathbf{u}\|_{C(\Omega)}\left(K h|\mathbf{u}|_{2, \Omega}+2\left\|\omega-C_{b} u^{(1)}\right\|_{A^{\prime}}+2\left\|C_{b} \varepsilon\right\|_{A^{\prime}}\right) .
\end{aligned}
$$

For the second term we have

$$
\begin{aligned}
\left|I_{2}\right| & =\left|\sum_{\sigma_{j} \in \bar{\Omega}} \varepsilon_{j} \frac{1}{h} \int_{\sigma_{j}}\left(\mathbf{u} \cdot \mathbf{t}-u_{j}^{t}\right)\left(M \omega^{\prime}\right)_{j} d s h h_{j}^{\prime}\right| \\
& \leq\left\|M \omega^{\prime}\right\|_{W}\|\varepsilon\|_{W, 4}\left(\sum_{\sigma_{j} \in \bar{\Omega}}\left(\frac{1}{h} \int_{\sigma_{j}}\left(\mathbf{u} \cdot \mathbf{t}-u_{j}^{t}\right) d s\right)^{4} h h_{j}^{\prime}\right)^{1 / 4} .
\end{aligned}
$$

Referring to the tangential components as defined by (8), we introduce

$$
\bar{u}_{j}^{t}:=\frac{1}{4}\left(\tilde{u}_{j_{a}}^{(1)}+\tilde{u}_{j_{b}}^{(1)}+\tilde{u}_{j_{c}}^{(1)}+\tilde{u}_{j_{d}}^{(1)}\right),
$$

where the tilde has the same meaning as in (8). Then we have

$$
\begin{aligned}
\left(\sum_{\sigma_{j} \in \bar{\Omega}}\left(\frac{1}{h} \int_{\sigma_{j}}\left(\mathbf{u} \cdot \mathbf{t}-u_{j}^{t}\right) d s\right)^{4} h h_{j}^{\prime}\right)^{1 / 4} \leq & \left(\sum_{\sigma_{j} \in \bar{\Omega}}\left(\frac{1}{h} \int_{\sigma_{j}}\left(\mathbf{u} \cdot \mathbf{t}-\bar{u}_{j}^{t}\right) d s\right)^{4} h h_{j}^{\prime}\right)^{1 / 4} \\
& +\left(\sum_{\sigma_{j} \in \bar{\Omega}}\left(\frac{1}{h} \int_{\sigma_{j}}\left(\bar{u}_{j}^{t}-u_{j}^{t}\right) d s\right)^{4} h h_{j}^{\prime}\right)^{1 / 4} .
\end{aligned}
$$

It follows from standard approximation theory that

$$
\left(\sum_{\sigma_{j} \in \bar{\Omega}}\left(\frac{1}{h} \int_{\sigma_{j}}\left(\mathbf{u} \cdot \mathbf{t}-\bar{u}_{j}^{t}\right) d s\right)^{4} h h_{j}^{\prime}\right)^{1 / 4} \leq K h\|\mathbf{u}\|_{2, \Omega},
$$

where $K$ is a constant independent of $h$. From (9) we have

$$
\begin{aligned}
\left(\sum_{\sigma_{j} \in \bar{\Omega}}\left(\frac{1}{h} \int_{\sigma_{j}}\left(\bar{u}_{j}^{t}-u_{j}^{t}\right) d s\right)^{4} h h_{j}^{\prime}\right)^{1 / 4} & =\left\|\bar{u}^{t}-u^{t}\right\|_{W, 4} \\
& \leq\left\|u^{(1)}-u\right\|_{W, 4} \\
& \leq \sigma\left\|C_{b} \varepsilon\right\|_{A^{\prime}}
\end{aligned}
$$

We now have the estimate for $I_{2}$ :

$$
\begin{aligned}
\left|I_{2}\right| & \leq\left\|M \omega^{\prime}\right\|_{W}\|\varepsilon\|_{W, 4}\left(K h\|\mathbf{u}\|_{2, \Omega}+\sigma\left\|C_{b} \varepsilon\right\|_{A^{\prime}}\right) \\
& \leq 2 \sigma\left\|\omega^{\prime}\right\|_{A^{\prime}}\left\|C_{b} \varepsilon\right\|_{A^{\prime}}\left(K h\|\mathbf{u}\|_{2, \Omega}+\sigma\left\|C_{b} \varepsilon\right\|_{A^{\prime}}\right)
\end{aligned}
$$


and the estimate for the nonlinear term becomes

$$
\begin{aligned}
\left|\left(\varepsilon, Z-Z^{\prime}\right)\right| \leq & \sigma\left\|C_{b} \varepsilon\right\|_{A^{\prime}}\|\mathbf{u}\|_{C(\Omega)}\left(K h|\mathbf{u}|_{2, \Omega}+2\left\|\omega-C_{b} u^{(1)}\right\|_{A^{\prime}}+2\left\|C_{b} \varepsilon\right\|_{A^{\prime}}\right) \\
& +2 \sigma\left\|C_{b} \varepsilon\right\|_{A^{\prime}}\left\|\omega^{\prime}\right\|_{A^{\prime}}\left(K h\|\mathbf{u}\|_{2, \Omega}+\sigma\left\|C_{b} \varepsilon\right\|_{A^{\prime}}\right) \\
= & \left.2 \sigma\left\|C_{b} \varepsilon\right\|_{A^{\prime}}^{2}\|\mathbf{u}\|_{C(\Omega)}+\sigma\left\|\omega^{\prime}\right\|_{A^{\prime}}\right)+K h\left\|C_{b} \varepsilon\right\|_{A^{\prime}}\|\mathbf{u}\|_{2, \Omega}\left\|\omega^{\prime}\right\|_{A^{\prime}} \\
& +K h\left\|C_{b} \varepsilon\right\|_{A^{\prime}}\|\mathbf{u}\|_{2, \Omega}\|\mathbf{u}\|_{C(\Omega)}+2 \sigma\left\|C_{b} \varepsilon\right\|_{A^{\prime}}\|\mathbf{u}\|_{C(\Omega)}\left\|\omega-C_{b} u^{(1)}\right\|_{A^{\prime}} .
\end{aligned}
$$

Hence,

$$
\begin{aligned}
\nu\left\|C_{b} \varepsilon\right\|_{A^{\prime}} \leq & \left\|C_{b} \varepsilon\right\|_{A^{\prime}}\left(2 \sigma\|\mathbf{u}\|_{C(\Omega)}+2 \sigma^{2}\left\|\omega^{\prime}\right\|_{A^{\prime}}\right)+\sigma\left\|\mu\left(\frac{\partial p}{\partial n}\right)-G \bar{p}\right\|_{W} \\
& +\left(\nu+2 \sigma\|\mathbf{u}\|_{C(\Omega)}\right)\left\|\omega-C_{b} u^{(1)}\right\|_{A^{\prime}}+K h\|\mathbf{u}\|_{2, \Omega}\left(\|\mathbf{u}\|_{C(\Omega)}+\left\|\omega^{\prime}\right\|_{A^{\prime}}\right),
\end{aligned}
$$

which leads to

$$
\left\|C_{b} \varepsilon\right\|_{A^{\prime}} \leq \frac{K h}{\nu(1-\bar{\sigma})}+\frac{K}{\nu(1-\bar{\sigma})}\left(\left\|\omega-C_{b} u^{(1)}\right\|_{A^{\prime}}+\left\|\mu\left(\frac{\partial p}{\partial n}\right)-G \bar{p}\right\|_{W}\right),
$$

where

$$
\bar{\sigma}=\frac{4 \sigma^{3}}{\nu^{2}}\|f\|_{W}+\frac{2 \sigma\|\mathbf{u}\|_{C(\Omega)}}{\nu}<1 .
$$

By standard approximation theory, we have

$$
\left\|\mu\left(\frac{\partial p}{\partial n}\right)-G \bar{p}\right\|_{W} \leq K h|p|_{2, \Omega}
$$

Define $u^{(2)}$ and $\bar{\omega}$ by

$$
u_{j}^{(2)}:=\frac{1}{h} \int_{\sigma_{j}^{\prime}} \mathbf{u} \cdot \mathbf{n} d s, \quad \bar{\omega}_{k}:=\frac{1}{A_{k}^{\prime}} \int_{\tau_{k}^{\prime}} \omega, \quad \nu_{k} \in \Omega,
$$

and follow the remark after [5, Theorem 6.1]; we have

$$
\begin{aligned}
\left\|\bar{\omega}-C_{b} u^{(1)}\right\|_{A^{\prime}} & \leq K h^{-1}\left\|u^{(2)}-u^{(1)}\right\|_{W} \\
& \leq K h\|\mathbf{u}\|_{2, \Omega} .
\end{aligned}
$$

Therefore, by standard approximation theory, we get

$$
\begin{aligned}
\left\|\omega-C_{b} u^{(1)}\right\|_{A^{\prime}} & \leq\|\omega-\bar{\omega}\|_{A^{\prime}}+\left\|\bar{\omega}-C_{b} u^{(1)}\right\|_{A^{\prime}} \\
& \leq K h|\mathbf{u}|_{2, \Omega} .
\end{aligned}
$$

Finally, we have the vorticity error estimate

$$
\begin{aligned}
\left\|\omega^{\prime}-\bar{\omega}\right\|_{A^{\prime}} & \leq\left\|C_{b} \varepsilon\right\|_{A^{\prime}}+\left\|\bar{\omega}-C_{b} u^{(1)}\right\|_{A^{\prime}} \\
& \leq K(\mathbf{u}, p, \mathbf{f}, \nu) h,
\end{aligned}
$$

where $K$ depends on $\|\mathbf{u}\|_{2, \Omega},|p|_{2, \Omega},\|f\|_{W}$ and $\nu$ but not on $h$. This finishes the proof of Theorem 2. 


\section{Pressure error estimate}

We begin by recalling the following standard result:

Lemma 4. The equation

$$
\operatorname{div} \mathbf{v}=f \in L_{0}^{2}(\Omega)
$$

has a solution $\mathbf{v} \in \mathbf{H}_{0}^{1}(\Omega)$ satisfying

$$
\|\mathbf{v}\|_{1, \Omega} \leq K\|f\|_{0, \Omega} .
$$

Proof. The proof of this lemma may be found in [2, p. 22].

We will apply this result with

$$
f:=\bar{p}-p^{\prime} \in L_{0}^{2}(\Omega),
$$

where the right side denotes the piecewise constant function with these values in each cell. Clearly,

$$
\left\|\bar{p}-p^{\prime}\right\|_{0, \Omega}=\left\|\bar{p}-p^{\prime}\right\|_{A}
$$

so that

$$
\|\mathbf{v}\|_{1, \Omega} \leq K\left\|\bar{p}-p^{\prime}\right\|_{A} .
$$

Next, we introduce $v^{(1)}$ and $v^{*}$ defined as follows:

$$
\begin{array}{rlrl}
\left.v^{(1)}\right|_{\sigma_{j}} & :=\frac{1}{h} \int_{\sigma_{j}} \mathbf{v} \cdot \mathbf{n} d s, & & \sigma_{j} \in \bar{\Omega}, \\
\left.v^{*}\right|_{\sigma_{j}^{\prime}}:=\frac{1}{h} \int_{\sigma_{j}^{\prime}} \mathbf{v} \cdot \mathbf{n} d s, & \sigma_{j}^{\prime} \in \bar{\Omega} .
\end{array}
$$

Use of the divergence theorem shows that

$$
D v^{(1)}=\bar{p}-p^{\prime} .
$$

In addition, we have

$$
\left\|v^{(1)}\right\|_{W} \leq K\|\mathbf{v}\|_{1, \Omega} \leq K\left\|\bar{p}-p^{\prime}\right\|_{A} .
$$

Only the first inequality is new. To prove it, consider the linear functional

$$
B \mathbf{v}:=\frac{1}{h} \int_{\sigma_{j}} \mathbf{v} \cdot \mathbf{n} d s .
$$

By mapping $\kappa_{j}$ to a standard kite $\hat{\kappa}$, we have that $B \mathbf{v}$ is bounded on $\mathbf{H}^{1}(\hat{\kappa})$. Then mapping $\hat{\kappa}$ back to $\kappa_{j}$ gives the result.

Now we have

$$
\left(C_{b} v^{*}\right)_{k}=\frac{1}{A_{k}^{\prime}} \int_{\partial A_{k}^{\prime}} \mathbf{v} \cdot \mathbf{t} d s=\frac{1}{\tau_{k}^{\prime}} \int_{\tau_{k}^{\prime}} \operatorname{curl} \mathbf{v} d x d y,
$$

from which it follows that

$$
\left\|C_{b} v^{*}\right\|_{A^{\prime}}^{2} \leq\|\operatorname{curl} \mathbf{v}\|_{0, \Omega}^{2} \leq\|\mathbf{v}\|_{1, \Omega}^{2} \leq K\left\|\bar{p}-p^{\prime}\right\|_{A}^{2} .
$$

We will need an estimate for $\left\|C_{b} v^{(1)}\right\|_{A^{\prime}}$. To obtain it, we first note the estimate

$$
\left\|v^{*}-v^{(1)}\right\|_{W} \leq K h\|\mathbf{v}\|_{1, \Omega} .
$$


The proof of this is given in [5]. Then

$$
\begin{aligned}
\left\|C_{b} v^{(1)}\right\|_{A^{\prime}} & \leq\left\|C_{b}\left(v^{*}-v^{(1)}\right)\right\|_{A^{\prime}}+\left\|C_{b} v^{*}\right\|_{A^{\prime}} \\
& \leq \frac{K}{h}\left\|v^{*}-v^{(1)}\right\|_{W}+\left\|C_{b} v^{*}\right\|_{A^{\prime}} \\
& \leq K\left\|\bar{p}-p^{\prime}\right\|_{A},
\end{aligned}
$$

where (21) was used.

Theorem 3. Under the assumptions of Theorems 1 and 2, we have the following estimate:

$$
\left\|p^{\prime}-\bar{p}\right\|_{A} \leq K(\mathbf{u}, p, \mathbf{f}, \nu) h,
$$

where $K$ depends on $\|\mathbf{u}\|_{2, \Omega},|p|_{2, \Omega},\|f\|_{W}$ and $\nu$ but not on $h$.

Proof. Taking the inner product of $v^{(1)}$ with the basic error equation gives

$$
\begin{array}{r}
\left(v^{(1)}, \nu R\left(\omega-\omega^{\prime}\right)\right)_{W}-\left(v^{(1)}, Z-Z^{\prime}\right)_{W}+\left(v^{(1)}, G\left(\bar{p}-p^{\prime}\right)\right)_{W} \\
=\left(v^{(1)}, G \bar{p}-\mu\left(\frac{\partial p}{\partial n}\right)\right)_{W} .
\end{array}
$$

Using summation by parts, we obtain

$$
\begin{aligned}
\left\|\bar{p}-p^{\prime}\right\|_{A}^{2} \leq & \nu\left\|C_{b} v^{(1)}\right\|_{A^{\prime}}\left\|\omega-\omega^{\prime}\right\|_{A^{\prime}}+\left\|v^{(1)}\right\|_{W}\left\|G \bar{p}-\mu\left(\frac{\partial p}{\partial n}\right)\right\|_{W} \\
& +\left|\left(v^{(1)}, Z-Z^{\prime}\right)_{W}\right| .
\end{aligned}
$$

For the nonlinear term, similarly to the estimates of $I_{1}$ and $I_{2}$, we get

$$
\begin{aligned}
\left|\left(v^{(1)}, Z-Z^{\prime}\right)\right| \leq & \left\|v^{(1)}\right\|_{W}\|\mathbf{u}\|_{C(\Omega)}\left(K h|\mathbf{u}|_{2, \Omega}+2\left\|\omega-C_{b} u^{(1)}\right\|_{A^{\prime}}+2\left\|C_{b} \varepsilon\right\|_{A^{\prime}}\right) \\
& +2\left\|v^{(1)}\right\|_{W, 4}\left\|\omega^{\prime}\right\|_{A^{\prime}}\left(K h\|\mathbf{u}\|_{2, \Omega}+\sigma\left\|C_{b} \varepsilon\right\|_{A^{\prime}}\right) .
\end{aligned}
$$

Using (20) and the approximation error estimates, we have

$$
\left|\left(v^{(1)}, Z-Z^{\prime}\right)\right| \leq K(\mathbf{u}, p, \mathbf{f}, \nu) h\left(\left\|v^{(1)}\right\|_{W}+\left\|v^{(1)}\right\|_{W, 4}\right) .
$$

To get a bound for $\left\|v^{(1)}\right\|_{W, 4}$, we introduce $\bar{v} \in \mathcal{U}$ defined on each $\kappa_{j}$ by

$$
\left.\bar{v}\right|_{\kappa_{j}}:=\frac{1}{\left|\kappa_{j}\right|} \int_{\kappa_{j}} \mathbf{v} \cdot \mathbf{n} d x d y .
$$

Clearly, $v_{j}^{(1)}-\bar{v}_{j}$ is bounded on $\mathbf{H}^{1}(\hat{\kappa})$ and vanishes for constant functions. Then

$$
\left|v_{j}^{(1)}-\bar{v}_{j}\right| \leq K|\mathbf{v}|_{1, \kappa_{j}},
$$

and therefore

$$
\begin{aligned}
\left\|v^{(1)}-\bar{v}\right\|_{W, 4} & \leq K\left(\sum_{j}|\mathbf{v}|_{1, \kappa_{j}}^{4} h h_{j}^{\prime}\right)^{1 / 4} \\
& \leq K h^{1 / 2}\left(\sum_{j}|\mathbf{v}|_{1, \kappa_{j}}^{4}\right)^{1 / 4} \\
& \leq K h^{1 / 2}|\mathbf{v}|_{1, \Omega} .
\end{aligned}
$$


On the other hand,

$$
\left|\bar{v}_{j}\right|=\left|\frac{1}{|\hat{\kappa}|} \int_{\hat{\kappa}} \hat{\mathbf{v}} \cdot \hat{\mathbf{n}} d \hat{x} d \hat{y}\right| \leq K|\hat{\mathbf{v}}|_{0,4, \hat{\kappa}}
$$

and therefore

$$
\begin{aligned}
\|\bar{v}\|_{W, 4} & \leq K h^{-1 / 2}\left(\sum_{j}|\mathbf{v}|_{0,4, \kappa_{j}}^{4} h h_{j}^{\prime}\right)^{1 / 4} \\
& \leq K|\mathbf{v}|_{0,4, \Omega} \\
& \leq K\|\mathbf{v}\|_{1, \Omega} .
\end{aligned}
$$

Hence, we have

$$
\begin{aligned}
\left\|v^{(1)}\right\|_{W, 4} & \leq\left\|v^{(1)}-\bar{v}\right\|_{W, 4}+\|\bar{v}\|_{W, 4} \\
& \leq K\|\mathbf{v}\|_{1, \Omega} \\
& \leq K\left\|\bar{p}-p^{\prime}\right\|_{A} .
\end{aligned}
$$

Then for the nonlinear term, we get

$$
\left|\left(v^{(1)}, Z-Z^{\prime}\right)\right| \leq K(\mathbf{u}, p, \mathbf{f}, \nu) h\left\|\bar{p}-p^{\prime}\right\|_{A} .
$$

Using the estimate for the vorticity and the approximation error estimates, we get the estimate for pressure,

$$
\left\|p^{\prime}-\bar{p}\right\|_{A} \leq K(\mathbf{u}, p, \mathbf{f}, \nu) h
$$

where $K$ depends on $\|\mathbf{u}\|_{2, \Omega},|p|_{2, \Omega},\|f\|_{W}$ and $\nu$ but not on $h$.

\section{ACKNOWLEDGMENT}

We would like to thank a referee for some helpful suggestions.

\section{REFERENCES}

1. N. Baba and H. Miyata, Numerical study of the three dimensional separating flow about obstacles with sharp corners, 11th Internat. Conf. Numer. Methods Fluid Dynamics (D. L. Dwoyer et al., eds.), Lecture Notes in Phys., vol. 323 Springer-Verlag, Berlin and New York, 1989 pp. $126-131$.

2. V. Girault and P.-A. Raviart, Finite element methods for Navier-Stokes equations, SpringerVerlag, Berlin and New York, 1986. MR 88b:65129

3. R. A. Nicolaides, Flow discretization by complementary volume techniques, AIAA Paper 891978, Proc. 9th AIAA meeting, Buffalo, New York, June, 1989.

4. - Analysis and convergence of the MAC scheme. I. The linear problem, SIAM J. Numer. Anal. 29 (1992), 1579-1591. MR 93j:65143

5. _ Direct discretization of planar div-curl problems, SIAM J. Numer. Anal. 29 (1992), 32-56. MR 93b:65176

6. R. A. Nicolaides and X. Wu, Numerical solution of the Hamel problem by a covolume method, Advances in Computational Fluid Dynamics (W. G. Habashi and M. Hafez,eds.), Gordon and Breach, 1995.

7. T. Porsching, Error estimates for MAC-like approximations to the linear Navier-Stokes equations, Numer. Math. 29 (1978), 291-306. MR 57:11348

Department of Mathematics, Carnegie Mellon University, Pittsburgh, Pennsylvania 15213

Current address, (X. Wu): Department of Mathematics, Hong Kong Baptist University, Kowloon, Hong Kong

E-mail address, X. Wu: xwu@hkbu.edu.hk 